\title{
OVERALL SURVIVAL INCREASE AFTER TREATMENT OF BRAIN METASTASIS FOR HER-2 POSITIVE PATIENTS USING TRASTUZUMAB
}

Nathalya A. Yagi', Nilceana M. A. Freitas', Thais F. Simionatto', Sylvia S. Pires', Mattheus H. Vale1, Jean T. Paiva', Edesio Martins ${ }^{1}$, Ruffo Freitas-Junior ${ }^{1}$

${ }^{1}$ Hospital Araújo Jorge, Centro Brasileiro de Radioterapia, Oncologia e Mastologia -Goiânia (GO), Brazil.

Objectives: Breast cancer is a heterogeneous, phenotypically diverse disease composed of several biological subtypes that have distinct behavior. Amplification or overexpression of the human epidermal growth factor receptor 2 (HER2) oncogene is presented in approximately $15 \%$ of primary invasive breast cancers. Target anti-HER 2 therapies have become important agents in the treatment of metastatic breast cancer and have altered the natural course of HER2-positive breast cancer. The risk of brain metastasis in women with overexpression HER2 is $12 \%$ in 10 years and a modality of treatment with brain radiosurgery (SRS) has been responsible for increasing local brain control with low morbidity. Methodology: Patients with diagnosis of breast cancer brain metastasis treated with SRS from 2007 to 2017 were divided into 3 groups: 1) HER2-positive who received treatment with trastuzumab (subgroup-1); 2) HER2-positive who didn't receive treatment with trastuzumab (subgroup-2) and; 3) HER2 negative, including triple negative and luminal (subgroup-3). Statistical analysis was performed using Fisher's test and Kaplan-Meier curve, considering 5 criteria separately: overall survival (OS), progression free survival (PFS) for the central nervous system (CNS) after radiosurgery, brain disease free survival (DFS) after diagnosis and survival after radiosurgery for brain metastasis (OSARS-overal survival after RS). Results: Among the 78 patients with brain metastasis of breast cancer treated with SRS, 37\% were HER2-positive. WBRT can be avoided in 66\% HER2-positive patients, and in the subgroup- 1 can be avoided in $62 \%$. OS in 5 years was $31 \%$ (mean: 34 months, 95\%CI 26.95-42.49). OS after diagnose CNS metastasis in 5 years was 18\% (95\%CI 26.95-42.49). PFS for the CNS after 1st radiosurgery in 1 year was $67 \%$ and 5 years was $9 \%$ (mean: 56months, $95 \% \mathrm{CI} 19.48-31.50$ ). The average time patient was alive after CNS metastasis was 56 months for subgroup-1 and 31 months for subgroup-2 and 36 months for subgroup-3. OS in 2 years after treatment of CNS metastasis was $80 \%$ for subgroup-1 (OR=0.9, 95\%CI 0.19-4.5, $\mathrm{p}=0.01$ ) when compared with subgroup-2, and OS in 2 years after treatment of CNS metastasis was $68 \%$ for subgroup-2 and $65 \%$ for subgroup-3. Conclusion: The results corroborate that SRS of CNS metastasis combined with trastuzumab therapy in HER2-positive patients may act in synergism, contributing with OS increase after treatment with SRS in CNS metastasis. 